\title{
Production of Equal Sized Atomic Clusters by a Hot Wire
}

\section{Peineke ${ }^{1}$, M. Attoui ${ }^{2}$, R. Robles ${ }^{3}$, A.C. Reber ${ }^{3}$, S.N. Khanna ${ }^{3}$, A. Schmidt-Ott ${ }^{1}$}

\author{
${ }^{1}$ Nanostructured Materials, DelftChemTech, Delft University of Technology, Julianalaan 136, NL-2628BL \\ Delft, The Netherlands \\ ${ }^{2}$ Département de Physique, Faculté des Sciences et Technologie, Université Paris XII, France \\ ${ }^{3}$ Department of Physics, Virginia Commonwealth University, Richmond, Virginia 23284.
}

\begin{abstract}
A resistively heated metal wire is shown to be a source of charged atomic clusters consisting of only a few atoms. They are size classified with a differential mobility analyzer, and their relative abundance is determined as a function of size. $\mathrm{Ag}_{\mathrm{n}} \mathrm{K}^{+}$clusters are obtained from wires containing silver with traces of potassium to provide the electric charge. First principles calculations reveal that the abundance observed can be fully explained by the energetic and chemical stability of the neutral cluster and $\mathrm{K}^{+}$attachment energy. $\mathrm{K}^{+}$attachment is a noninvasive way of charging, as the observed $\mathrm{Ag}_{\mathrm{n}} \mathrm{K}^{+}$cluster properties are similar with respect to the pure $\mathrm{Ag}_{\mathrm{n}}$ clusters in terms of energetic and electronic stability and cluster structure. The equally-sized clusters are basically available for reactivity, coalescence and deposition studies, and the method is extendable to other materials.
\end{abstract}

\section{Introduction:}

The field of atomic clusters continues to generate excitement largely due to two reasons. First, unexpected behaviors are found to emerge as the cluster size is reduced to sub-nanometer scale. For example, clusters of non-magnetic solids are found to be magnetic (Reddy, Khanna et al., 1993, Cox et al., 1994), while clusters of noble metals like gold are found to be strong catalysts (Heiz et al., 1999, Wallace et al., 2003 and Zhang et al., 2007). Further, the properties change with size and composition allowing tailoring of the properties as every atom counts in the cluster regime (Castleman, and Jena, 2006). Secondly, it has been found that using stable clusters, it may be possible to form cluster assembled materials where clusters serve as the building blocks (Khanna and Jena, 1992). The discovery of fullerenes (Kroto et al., 1985) and the formation of fullerides demonstrated this exciting possibility and over the past few years, several stable solids composed of $\mathrm{Au}_{102}$, (Jadzinsky et al., 2007), $\mathrm{Au}_{25}$ (Heaven et al., 2008), As 7 (Castleman et al., 2007), $\mathrm{Te}_{2} \mathrm{As}_{2}$ (Ugrinov et al., 2008) and even mixed cluster assemblies with $\mathrm{Au}_{7}$ and $\mathrm{Au}_{8}$ with $\mathrm{C}_{60}$ (Schultz-Dobrick et al., 2008) have been synthesized. This line of development is particularly exciting in view of the finding that certain stable species can mimic the chemical behavior of atoms in the periodic table and can be regarded as superatoms forming a new dimension of the periodic table (Khanna and Jena, 1995, Bergeron et al., 2004, Bergeron et al., 2005, Reber et al., 2007). As the properties of clusters can be changed by size and composition, this allows the possibility of synthesizing materials with controllable properties.

A major challenge in experimental studies on atomic clusters is to produce arbitrary pure and uniformly sized samples that are available for studies on chemical interaction and stability, and to allow surface deposition for production of cluster assembled materials. In view of the latter, increase of the production rate with respect to existing technology is another challenge. The concept of the superatom reveals that improvements in this area will undoubtedly lead to discovery of new materials with interesting properties, which will trigger process 
development for industrial scale production. Inert gas suspension in the mbar regime has been used for cluster reactivity studies since the early nineties, and mobility analysis in a drift cell was performed in those same studies (Kemper and Bowers, 1990, Manard et al., 2006) on mass separated clusters. While the combination of mass separation with mobility analysis proved to be a very powerful instrument for cluster research, this required coupling a pressurized system to vacuum systems on both sides. Sophisticated set-ups with differential pumping and upper pressure limits of a few mbar are applied. The material quantities that can be processed this way are small, and gated operation of the drift cell extracts short packets which only represent a small fraction of the clusters present, typically below $1 \%$. In the approach of the present paper, a differential mobility analyzer continuously selects all the clusters of a selected size, and the whole system is operated at the same pressure, which minimizes the loss of clusters. We estimate that the rate with which clusters of uniform size can be provided with our system is at least a factor 100 larger than the state of the art.

A preliminary study (Peineke and Schmidt-Ott, 2007) had shown that charged species emitted from a glowing wire produce spikes in the mobility distribution that were attributed to clusters of outstanding stability (magic numbers). Recent developments in improvement of normal pressure differential mobility classifiers have led to devices that separate ionic species in a gas with high resolution (Fernandez de la Mora, and Attoui, 2007). These devices have been applied to nanoparticles, gas ions and large molecules in the past (Fernández de la Mora et al., 1998, Saucy et al., 2004, Fernández de la Mora et al., 2006), but not to atomic clusters, in lack of a suitable source that also provides electrical charge.

One purpose of the present article is to demonstrate that an electrically heated wire is a very simple source of atomic clusters that can be purified via continuous mobility separation. Another purpose is to demonstrate that the electric charge required for the electrostatic separation step can be provided by the presence of traces of $\mathrm{K}$ in the wire material, and calculations show that this is rather non-invasive, having little influence on the neutral cluster properties.

\section{Experimental set-up}

Fig 1. shows the experimental set-up. The charged atomic clusters produced by the glowing wire are passed through a differential mobility analyzer. The gas with the selected species could exit the classifier, where it would be available for studies on chemical properties and stability. In the present experiment the selected species was simply precipitated to an electrode collecting the charge. The corresponding current was recorded as a function of the selected mobility, which yields the relative abundance of cluster sizes.

\subsection{Production of atomic clusters}

We recently studied the production of charged nanoparticles $(>2 \mathrm{~nm})$ from electrically heated metal wires in inert gases (Peineke et al. 2006, Peineke and Schmidt-Ott, 2008). The method is elegant, because only the wire gets hot, which avoids contaminants from other surfaces. Below the melting point, most metals exhibit a large enough vapor pressure to form nanoparticles from the condensing vapor. Evaporation is accompanied by thermal emission of electrons at high enough temperatures. A fraction of the particles produced then obtain an elementary charge through electron attachment. Again the source of this charge is the wire itself, avoiding introduction of contaminants, as generally produced by aerosol chargers if conditions are not completely clean (Fernandez de la Mora et al., 2003). At wire temperatures below the limit of observable thermal emission, positively charged particles are formed. We have explained this by alkali metal impurities (Peineke and Schmidt-Ott, 2008) and found that $\mathrm{K}+$ emission plays a dominant role, because $\mathrm{K}$ is present in virtually all commercial metals. It has a high probability of being emitted from the wire as a cation because its ionization energy 
is not much higher than the work function of the metal (Saha-Langmuir equation). The lower the temperature the smaller the particles that form, and for each metal we find a temperature zone, where atomic clusters are predominantly formed. We have realized that in this temperature zone the contribution of impurities that have a higher vapor pressure than the host metal may initially be dominant but can be outgassed. In the present study we concentrated on $\mathrm{Ag}$, and available $\mathrm{Ag}$ wires have almost no impurities that are more volatile than Ag. With this choice we are limited to the positive polarity, since thermal emission is negligible below the melting point of $\mathrm{Ag}$. $\mathrm{K}+$ ions are thus our charge source. This was confirmed by a mass spectrum below $300 \mathrm{amu}$, which showed some $\mathrm{K}^{+}$, and almost exclusively $\mathrm{Ag}_{2} \mathrm{~K}^{+}$. This is in agreement with the high stability of this cluster (see below) and confirms that our Ag wire is predominantly a source of $\mathrm{Ag}$ with $\mathrm{K}^{+}$as the dominant charged species. $\mathrm{Ag}$ and $\mathrm{K}$ are relatively volatile impurities present also in other commercial metal wires, and the $\mathrm{Ag}_{2} \mathrm{~K}^{+}$ peak was also predominant in the mass spectrum of clusters from a commercial Pd wire. The Ag impurity segregates to the surface, having the lower surface tension. This effect can be enhanced by wetting the wire with a thin layer of the metal(s) chosen to form the clusters, and, if necessary, the alkali metal to deliver the charge. Similarly, Au nanoparticles have been produced by wetting a W wire with $\mathrm{Au}$ (Burtscher, Schmidt-Ott et al., 1984). The wires used in the present study had purities of $99.995 \%$.

\subsection{Electrostatic Mobility Classification}

The mobility classifier concept is illustrated in fig. 1 . The model we applied has been described in a conference contribution (Fernandez de la Mora and Attoui, 2007) and in the meantime it is available from NanoEngineering Corp., Florida, USA. It resembles the concept of Fernandez de la Mora et al., 2006. The atomic clusters, carried by an inert gas, here $\mathrm{N}_{2}$ $(99.9995 \%)$, enter through an annular slit. This flow is separated from the center rod by a filtered (cluster-free) sheath gas. A variable voltage $\mathrm{V}$ between the inner rod and the outer cylinder produces a radial electric field that deflects the charged clusters towards the center rod. This velocity is superimposed on the laminar gas flow, and the selected cluster mobility exits through the slit in the center rod. Due to the funnel profile, the flow is strongly accelerated to reach a high velocity in the mobility separation zone, which keeps the corresponding time small enough (ca. $1 \mathrm{~ms}$ ) for tolerable diffusion broadening, which determines resolution. The necessary velocity corresponds to a Reynolds number in the turbulent range, but the time is too short for turbulence to develop. The selected mobility is defined by the radial velocity component $v$ induced by the field $\mathrm{E}$ via

$$
Z=\frac{v}{E} \text {. }
$$

With a given vertical flow velocity and geometry, the inverse mobility $\mathrm{Z}^{-1}$ is therewith related to the Voltage $\mathrm{V}$ applied between the inner rod and outer cylinder through

$$
Z^{-1}=\text { const } V
$$

We determine the constant by calibrating with tetraheptyl ammonium ions produced by electrospray of a $1 \mathrm{mM}$ solution in pure methanol, for which $Z=0.96 \mathrm{~cm}^{2} / \mathrm{V} / \mathrm{s}$ is assumed. We used filtered air as the rapidly consumed sheath gas $(1000 \mathrm{~L} / \mathrm{min})$ for cost reasons and because any etching or attachment of molecules during the short deflection period of ca. $1 \mathrm{~ms}$ did not hinder atomic resolution in the separation of cluster sizes. For particles much smaller than the mean free path of the gas molecules it has been verified by Fernandez de la Mora et al. (2003) that the inverse mobility is proportional to the square of the collision diameter of the atomic cluster, 
$Z^{-1} \propto\left(d_{c l}+d\right)^{2}$

Here $d_{c l}+d$ represents the collision diameter, where $d_{c l}$ is the cluster diameter and $\mathrm{d}$ accounts for the diameter of the gas molecules. The relation above neglects polarization and recoil effects (Tammet, 1995) and is a rather crude approximation for particles in the ionic or atomic cluster size range. It is sufficient for the present purpose, though, because we apply it here only to show consistency of our mobility spectra with discrete cluster sizes differing in the number of atoms. The Ag-Ag and the $\mathrm{Ag}-\mathrm{K}^{+}$bond lengths calculated are different, but we use the simplifying assumption that all atoms occupy the same volume. Further simplifying by saying that a cluster is a spherical body, the volume of which is the sum of the atomic volumes, we assign the diameter

$d_{c l}=d_{a t}(n+1)^{1 / 3}$

to a $\mathrm{Ag}_{\mathrm{n}} \mathrm{K}^{+}$cluster, with $d_{a t}$ representing the atomic diameter. Eqs. (3) and (4) lead to the relation

$Z^{-1}=c\left(\frac{d_{a t}}{d}(n+1)^{1 / 3}+1\right)^{2}$,

where $\mathrm{c}$ is a constant depending on temperature, pressure and type of gas.

\section{Cluster Stability Calculations}

First principles electronic structure calculations were carried out to probe the relative stability of the $\mathrm{Ag}_{\mathrm{n}}$ clusters and the effect of $\mathrm{K}^{+}$on the properties of pure clusters. The theoretical investigations focused on the ground state geometry, stability, and electronic properties of neutral and cationic $\mathrm{Ag}_{\mathrm{n}}$, and $\mathrm{KAg}_{\mathrm{n}}$ clusters containing up to 7 atoms. We used an allelectron linear combination of atomic orbitals molecular orbital approach within a density functional framework. The exchange correlation effects were included via a gradient corrected functional (Perdew et al., 1996). The molecular orbitals were expressed as a linear combination of Gaussian type orbitals centered at the atomic sites. The actual calculations were carried out using the NRLMOL set of codes developed by Pederson and co-workers (Pederson and Jackson, 1990, Jackson and Pederson, 1990, Porezag and Pederson, 1999). The basis sets for Ag had 8s, 6p, and 5d Gaussians while those of $\mathrm{K}$ had 7s, 5p, and 3d functions. The basis sets were augmented by additional Gaussians. The ground state geometries were obtained by starting from several initial configurations and moving atoms in the direction of forces till the forces dropped below a threshold value. Various spin configurations were tried to ensure that the ground state is optimized with respect to geometry as well as spin multiplicity.

Fig. 2a shows the ground state geometries of pure $\mathrm{Ag}_{\mathrm{n}}$ clusters containing up to $7 \mathrm{Ag}$ atoms and Fig. $2 \mathrm{~b}$ shows the respective geometries with the $\mathrm{K}^{+}$cation added. For each cluster, we also calculated the gap between the highest occupied molecular orbital (HOMO) - and the lowest unoccupied molecular orbital (LUMO). The HOMO-LUMO gap (H-L gap) is a measure of the chemical stability of the systems as clusters with large HOMO-LUMO gaps neither prefer to donate charge nor receive charge, and are unlikely to undergo electronic excitations (Pearson, 1986, Reber et al., 2007, Roach et al., 2007). We also calculated the 
ionization potential (IP) representing the difference in energy between the ground state of the neutral and the cation. H-L gap and IP are shown in Fig. 3.

Fig. 2a shows that, for neutral clusters, the ground state structures are planar up until 6 atoms, and for $\mathrm{Ag}_{7}$ it is a pentagonal pyramid. These findings are in agreement with earlier calculations by Fournier (2001) and Fernandez et al. (2004) . Comparing the structures of $\mathrm{Ag}_{\mathrm{n}}$ and $A g_{n}-K^{+}$in Fig. $2 b$, we see that the ground state $A g_{n}$ structures are hardly changed by $\mathrm{K}^{+}$ attachment with the exception of $n=4$. The electronic properties are represented by the H-L gap and the IP value and plotted vs. cluster size in Fig. 3. Figs. 2 and 3 show that the influence of $\mathrm{K}^{+}$attachment on the electronic properties is also small. In particular, the evenodd oscillations vs. size in the H-L gap are retained. They are due to electron pairing in the Ag clusters, and indicate enhanced chemical stability for even numbers of Ag atoms. We conclude that, in general, charging by $\mathrm{K}^{+}$attachment causes only a limited distortion of the $\mathrm{Ag}_{\mathrm{n}}$ clusters. This is also evident from the fact that the values of the IP are clearly higher than the IP of the $\mathrm{K}$ atom $(4.34 \mathrm{eV})$. In contrast, the even-odd oscillations are reversed for pure $\mathrm{Ag}_{\mathrm{n}}{ }^{+}$clusters compared to neutral species, as also shown in Fig. 3. This is because Ag atoms have one valence s electron, such that $\mathrm{Ag}_{\mathrm{n}}{ }^{+}$and $\mathrm{Ag}_{\mathrm{n}-1}$ have the same effective electron count.

We also calculated the removal energies (RE) defined as the energy required to remove a single atom from the cluster. This is also the energy gain in adding the respective atom to form the cluster from the preceding size. The calculated values of the $\mathrm{Ag}-\mathrm{Ag} \mathrm{g}_{\mathrm{n}-1}, \mathrm{Ag}-\mathrm{Ag}_{\mathrm{n}-1} \mathrm{~K}^{+}$ REs are given in Fig. 4. They show the same even-odd oscillations as the electronic properties in Fig. 3. So the even-n clusters are more stable, both in terms of energetic and chemical stability, with or without $\mathrm{K}^{+}$attached. For interpretation of the relative abundances experimentally observed, the $\mathrm{Ag}_{\mathrm{n}}-\mathrm{K}^{+} \mathrm{RE}$ (also in fig. 4) is important as well, since $\mathrm{K}^{+}$makes the clusters detectable. In addition, the $\mathrm{Ag}_{\mathrm{n}}{ }^{+}-\mathrm{K} \mathrm{RE}$ is plotted. Since it is much higher than the $\mathrm{Ag}_{\mathrm{n}}-\mathrm{K}^{+} \mathrm{RE}$, a $\mathrm{Ag}_{\mathrm{n}} \mathrm{K}^{+}$cluster will never split off a neutral $\mathrm{K}$, and the occurrence of $\mathrm{Ag}_{\mathrm{n}}{ }^{+}$ clusters can be excluded.

\section{Experimental Results and Interpretation}

The charged size selected clusters produce an electrometer current between $10^{-14}$ and $10^{-13} \mathrm{~A}$, corresponding to concentrations between $10^{3}$ and $10^{4} \mathrm{~cm}^{-3}$. Fig. 5 shows the cluster distribution produced with a silver wire in terms of the cluster current I as function of the inverse mobility selected by the DMA. We attribute the peaks to $\operatorname{Ag}_{\mathrm{n}} \mathrm{K}^{+}(n \geq 0)$ and assign the first prominent one to the smallest stable cluster, which is $\mathrm{Ag}_{2} \mathrm{~K}^{+}$. According to eq. $5, \mathrm{Z}^{-1}$ grows with the cluster size, and addition of single atoms leads to a series of peaks with decreasing distance for growing $\mathrm{n}$. This is what we observe in fig. 5 , and eq. 5 can be fitted to the peak positions fairly well (vertical lines), regarding the crudeness of the assumptions it is based on. The fit

yields $\frac{d_{a t}}{d}=1.8$ for the ratio of the metal atomic diameter and the gas molecule collision diameter. The latter is not available for $\mathrm{N}_{2}$-metallic cluster collisions, but the ratio would be expected smaller. The rather large value obtained is not surprising considering the large deviation of the $\mathrm{Ag}_{\mathrm{n}} \mathrm{K}^{+}$cluster structure from a volume equivalent packing (fig. 2). The clusters produced by the Pd wire containing $\mathrm{Ag}$ and $\mathrm{K}$ show perfect agreement with the silver wire curve, confirming that the peaks are also due to $\mathrm{Ag}_{\mathrm{n}} \mathrm{K}^{+}$, which had been shown only for $\mathrm{Ag}_{2} \mathrm{~K}^{+}$by mass spectroscopy. We attribute the first peak to the left (hardly seen in the $\mathrm{Ag}$ wire spectrum) to $\mathrm{K}^{+}$. Expectedly, this peak does not match the fit to eq. 5, among others due to polarization effects that become more important with decreasing size of the charged species. The mobility distribution reveals pronounced differences in the populations of the cluster sizes. These clearly reflect higher stability of clusters with an even number of $\mathrm{Ag}$ 
atoms. In fig. 5 this is clarified by joining the peaks of the even number clusters in the distribution from the $\mathrm{Pd}-\mathrm{Ag}-\mathrm{K}$ wire. The odd cluster peaks are always below these lines.

From the highest peak in fig. 5 the resolution of the mobility measurement for the present size range can be estimated in terms of the relative standard deviation $\sigma_{r}$. For $\mathrm{Z}^{-1}$ this value is about $2 \%$. From eqs. (3) and (5) it can be derived that this corresponds to a $\sigma_{r}$ of about $1 \%$ in $d_{c l}$ and about $3 \%$ in $\mathrm{n}$. The absolute standard deviation in $\mathrm{Z}^{-1}$ is about $20 \%$ of the difference between two adjacent cluster sizes that differ in one atom. This is good enough to select clusters of uniform size (same $n$ ) in the present size range.

To compare the result of fig. 5 with the calculated values in fig. 4, we consider that the initial Ag atom concentration in the vapor is at least 3 orders of magnitude higher than the $\mathrm{K}^{+}$ concentration and that the $\mathrm{K}^{+}$concentration is comparable to the $\mathrm{K}$ concentration ${ }^{\text {Error! Bookmark }}$ not defined. Thus the role of $\mathrm{K}$ can be neglected with respect to $\mathrm{Ag}$. Yet $\mathrm{K}^{+}$is important, because the charge delivered by this ion makes our clusters detectable. Two charged clusters will not aggregate, and $\mathrm{Ag}_{\mathrm{n}}{ }^{+}$formation is not possible (see above). Thus the following growth mechanisms must be considered:

(1) $\mathrm{Ag}_{\mathrm{n}}$ cluster formation and growth from the vapor

(2) $\mathrm{K}^{+}$ion attachment to $\mathrm{Ag}_{\mathrm{n}}$ clusters to form $\mathrm{Ag}_{\mathrm{n}} \mathrm{K}^{+}$,

(3) attachment of neutral $\mathrm{Ag}$ and to a smaller extent attachment of neutral $\mathrm{Ag}_{\mathrm{m}}$ to $\mathrm{Ag}_{\mathrm{n}} \mathrm{K}^{+}$.

The clusters continue to grow until size classified. We conclude from the fact that unattached

$\mathrm{K}^{+}$as well as the unstable $\mathrm{Ag}-\mathrm{K}^{+}$(binding energy $0.3 \mathrm{eV}=12 \mathrm{kT}$ ) are present in the mobility classifier that some atomic Ag must be present until mobility separation occurs. This emphasizes the importance of step (3). The relative abundance of the clusters is thus determined by the binding energies associated with the steps (1) - (3), namely the removal energies of (1) $\mathrm{Ag}-\mathrm{Ag}_{\mathrm{n}-1}$, (2) $\mathrm{Ag}_{\mathrm{n}}-\mathrm{K}^{+}$and (3) $\mathrm{Ag}-\mathrm{Ag}_{\mathrm{n}-1} \mathrm{~K}^{+}$, plotted vs. $\mathrm{n}$ in fig. 4. The higher abundance of the $\mathrm{Ag}_{\mathrm{n}}-\mathrm{K}^{+}$clusters with even $\mathrm{n}$ in fig. 5 is in agreement with the calculations for $\mathrm{Ag}-\mathrm{Ag}_{\mathrm{n}-1}$ and with those for $\mathrm{Ag}-\mathrm{Ag}_{\mathrm{n}-1} \mathrm{~K}^{+}$. Since only charged clusters are visible, $\mathrm{K}^{+}$ removal energies from $\mathrm{Ag}_{\mathrm{n}}-\mathrm{K}^{+}$in fig. 5 are also important. This could probably account for the higher intensity of $\mathrm{Ag}_{4} \mathrm{~K}^{+}$over $\mathrm{Ag}_{2} \mathrm{~K}^{+}$as $\mathrm{K}^{+}$binds more strongly to $\mathrm{Ag}_{4}$ than to $\mathrm{Ag}_{2}$. They level off at $\mathrm{n}=3$ and do not introduce any structure. Summarizing, the relative cluster abundance should be dominated by the stabilities of $\mathrm{Ag}_{\mathrm{n}}$, and this is what the experiment shows.

We would like to add that the $\mathrm{Ag}_{\mathrm{n}} \mathrm{K}^{+}$clusters with even numbers of $\mathrm{Ag}$ atoms have HOMOLUMO gaps in excess of $1.0 \mathrm{eV}$. As we recently showed, clusters with such large HOMOLUMO gaps are protected against etching by oxygen due to spin effects. Hence even-n $\mathrm{Ag}_{\mathrm{n}} \mathrm{K}^{+}$ clusters should also be chemically stable against strong etchants. Etching with oxygen would thus lead to further discrimination of the odd-n clusters. Etching by the $\mathrm{O}_{2}$ in the sheath gas during the $1 \mathrm{~ms}$ separation period would lead to smearing of the odd and even number peaks. Due to the presence and sharpness of the odd-n peaks we conclude that etching did not have any significant influence on the relative abundance reflected by our mobility spectra.

\section{Conclusions}

We successfully showed that equally sized atomic clusters can be produced by combination of a resistively heated wire with differential mobility classification. Our study thus demonstrates that atomic clusters or superatoms pure in composition can be produced and studied by very simple means. With the mobility classifier applied it would be possible to extract uniformly sized particles in an inert gas flow for further studies. Concentrations 
presently achievable are between $10^{3}$ and $10^{4} \mathrm{~cm}^{-3}$. The production rate exceeds those of the state of the art by at least a factor of 100 . While the choice of $\mathrm{Ag}_{n} \mathrm{~K}^{+}$clusters was determined by wire materials readily available, it is clear that other cluster species could easily be produced by the same principle. As demonstrated with the Pd wire, any coated hot metal surface could be used to produce clusters of almost any other species or mixture of species of higher vapor pressure. The charge required for classification is obtained as a consequence of small concentrations of $\mathrm{K}$. Charging by doping with $\mathrm{K}$ is thus a general recipe for obtaining charged clusters, and it is of special interest, because $\mathrm{K}^{+}$attachment is not very invasive in the sense that disturbance of the neutral cluster properties is rather limited due to its noble gas character. In contrast, removing an electron from the cluster leads to completely different properties.

There strong correlation between the relative cluster abundance and the calculated binding energies, particularly reflected by the odd-even effect. This supports our interpretation of the measured mobility spectra as well as the model applied for the calculation. For the present study, which was not carried out with ultra pure gases, it demonstrates that possible contaminants in the gas play a minor role. The fact that the wire is both the source of the atomic cluster material and the charged species $\mathrm{K}^{+}$guarantees a much higher probability of charge transfer to the clusters than to any gaseous contaminant in the inert gas.

From its principle, the method demonstrated is extremely flexible with respect to material, cluster size range and cluster production rate. It will greatly expand the possibilities of studying atomic clusters and superatoms in the future. Inert gas suspension at normal pressure enables access to the clusters during a long time, and besides chemical reactions, coalescence behavior could be studied, which gives an indication of cluster stability in view of formation of cluster assembled materials. Electron attachment or thermal separation from the weakly bound alkali cation could be used to study neutralized clusters. The charging necessary for mass or mobility analysis of the product could apply charging techniques provided by aerosol technology such as photoelectron emission (Jung et al., 1988). Energetic cluster stability is important in view of cluster assembled materials, and thermal cluster fragmentation as well as coalescence could easily be studied in gas suspension as well as chemical reaction with gases or other clusters. A second mobility analyzer and/or inertial impaction (Fernandez de la Mora et al., 2003) and/or mass spectroscopy would form a powerful combination for analysis of the product.

\section{Acknowledgement}

We are grateful to Juan Fernandez de la Mora, who allowed experiments in his lab that were essential for the present study, in particular the use of his mass spectrometer. CP is grateful to DFG, since part of the study was based on results obtained in the frame of SFB445. RR, ACR and SNK are grateful to U. S. Department of Army (Multidisciplinary University Research Initiative Grant W911NF-06-1-0280).

\section{References}

Bergeron D.E., Castleman A.W., Morisato T., \& Khanna S.N. Formation of $\mathrm{Al}_{13} \mathrm{I}^{-}$: Evidence for the superhalogen character of $\mathrm{Al}^{-}$13. Science, 30484 (2004).

Bergeron D.E., Roach P.J., Castleman A.W., Jones N.O., \& Khanna S.N. (2005). Al cluster superatoms as halogens in polyhalides and as alkaline earths in iodide salts. Science, 307 231-235.

Castleman A.W., \& Jena P. (2006). Clusters: A bridge between disciplines. Proc. Nat. Acad. Sci. USA, 103 10552. 
Castleman Jr. A.W., Khanna S.N., Sen A., Reber A.C., Qian M., Davis K.M., Peppernick S.J., Ugrinov A., \& Meritt M.D. (2007). From Designer Clusters to Synthetic Crystalline Nanoassemblies. Nano Letters, 7, 2734. Cox, A.J. Louderback, J.G. \& Bloomfield L.A. (1994). Experimental observation of magnetism in rhodium clusters. Phys. Rev. Lett., 71, 923.

Fernandez de la Mora J., \& Attoui, M. (2007). Abstract T02A029 European Aerosol Conference 2007, Salzburg, Austria.

Fernández de la Mora J., de Juan L., Eichler T. \& Rosell J. (1998). Differential mobility analysis of molecular ions and nanometer particles. Trends in Anal. Chem., 17, 328-339.

Fernández de la Mora J., Ude S., \& Thomson B.A. (2006). The potential of differential mobility analysis coupled to MS for the study of very large singly and multiply charged proteins and protein complexes in the gas phase. Biotechnol. J., 1, 988-997.

Fernandez de la Mora, J., Liedtke, L., \& Schmidt-Ott, A. (2003). Mass and size determination of nanometer particles by means of mobility analysis and focussed impaction. Journal of Aerosol Science, 34, 78-98.

Fernandez E.M., Soler J.M., Garzon I.L., \& Balbas L.C. (2004). Trends in the structure and bonding of noble metal clusters. Phys. Rev. B, 70, 165403.

Fournier R. (2001). J. Chem. Phys. 115, 2165.

Heaven M.W., Dass A., White P.S., Holt K.M., \& Murray R.W. (2008). Crystal Structure of the Gold Nanoparticle $\left[\mathrm{N}\left(\mathrm{C}_{8} \mathrm{H}_{17}\right)_{4}\right]\left[\mathrm{Au}_{25}\left(\mathrm{SCH}_{2} \mathrm{CH}_{2} \mathrm{Ph}\right)_{18}\right]$. J. Amer. Chem. Soc., 130, 3754.

Heiz, U. Sanchez A., Abbert S., \& Schneider W.D. (1999). When Gold Is Not Noble: Nanoscale Gold Catalysts J. Phys. Chem. A, 103, 9573.

Jackson K., \& Pederson M.R. (1990). Accurate forces in a local-orbital approach to the local-density approximation. Phys. Rev. B. 42, 3276.

Jadzinsky P.D., Calero G., Ackerson C.J., Bushnell D.A., \& Kornberg R.D. (2007). Structure of a Thiol Monolayer-Protected Gold Nanoparticle at $1.1 \AA$ Resolution. Science, 318, 5149.

Jung T., Burtscher H., \& Schmidt-Ott A. (1988). Multiple Charging of Ultrafine Aerosol Particles by Aerosol Photoemission. J. Aerosol Sci., 19 , 485.

Kemper P.R. \& Bowers M.T. (1990). A Hybrid Double-Focussing Mass Spectrometer - High-Pressure Drift

Reaction Cell to Study Thermal Energy Reactions of Mass-Selected Ions. J.A. Soc. Mass Spectrom., 1, 197-207. Khanna S. N. \& Jena P. (1992). Assembling crystals from clusters. Phys. Rev. Lett., 69, 1664.

Khanna S. N. \& Jena P. (1995). Atomic Clusters - Building Blocks for a New Class of Solids, Phys. Rev. B, $51,13705$.

Kroto H.W., Heath J.R., O’Brien S.C., Curl R.F., \& Smalley R.E.. C C60 Buckminsterfullerene. Nature 318,162 (1985).

Manard M.J., Kemper P.R., \& Bowers M.T. (2006). An Experimental and Theoretical Investigation into the BindingInteractions of Silver Cluster Cations with Ethylene and Propene. Int. J. Mass Spectrm., 249-250, 252262.

Pearson R.G. (1986). Absolute electronegativity and hardness correlated with molecular orbital theory. Proc. Nat. Acad. Sci. U.S.A., 83, 8440.

Pederson M.R., \& Jackson K.A. (1990). Variational mesh for quantum-mechanical simulations. Phys. Rev. B., 417453.

Peineke C., \& Schmidt-Ott A. (2007). Atomic Clusters produced by a Glowing Wire, Proc. PARTEC 2007, Nurnberg, Germany, 27.3. - 29.3.2007 (3-page abstract).

Peineke C., \& Schmidt-Ott A., Explanation of charged nanoparticle production from hot surfaces, J. Aerosol Sci. 39, 244-252 (2008).

Peineke C., Attoui M.B., \& Schmidt-Ott A. (2006). Using a glowing wire generator for production of charged, uniformly sized nanoparticles at high concentrations, J. Aersol. Sci. 371651.

Perdew J.P., Burke K., \& Ernzerhof M. (1996). Generalized Gradient Approximation Made Simple. Physical Review Letters, 77, 3865.

Porezag D., \& Pederson M.R. (1999). Optimization of Gaussian basis sets for density-functional calculations. Phys. Rev. A., 60, 2840.

Reber A.C., Khanna S.N., \& Castleman Jr. A.W. (2007). Superatom Compounds, Clusters, and Assemblies: Ultra Alkali Motifs and Architectures. J. Amer. Chem. Soc., 12910189.

Reber A.C., Khanna S.N., Roach P.J., Woodward W.H., \& Castleman A.W. (2007). Spin Accommodation and Reactivity of Aluminum Based Clusters with $\mathrm{O}_{2}$. J. Amer. Chem. Soc. 129, 16098.

Reddy, B.V., Khanna, S.N. \& Dunlap, B.I. (1993). Giant Magnetic-moments in 4D clusters. Phys. Rev. Lett., $70,3323$.

Roach P.J., Reber A.C., Woodward W.H., Khanna S.N., \& Castleman Jr. A.W. (2007). $\mathrm{Al}_{4} \mathrm{H}_{7}{ }^{-}$is a resilient building block for aluminum hydrogen cluster materials. Proc. Nat. Acad. Sci. U.S.A., 104, 14565.

Saucy D., Ude S., Lenggoro W.\& Fernandez de la Mora J., (2004). Mass analysis of water-soluble polymers by mobility measurement of charge-reduced electrosprays. Anal. Chem., 76,1045-1053. 
Schultz-Dobrick M., \& Jansens M(2008). Intercluster Compounds Consisting of Gold Clusters and Fullerides: $\left[\mathrm{Au}_{7}\left(\mathrm{PPh}_{3}\right)_{7}\right] \mathrm{C}_{60}-\mathrm{THF}$ and $\left[\mathrm{Au}_{8}\left(\mathrm{PPh}_{3}\right)_{8}\right]\left(\mathrm{C}_{60}\right)_{2}$. Angew. Chem., 47, 2256.

Tammet H. (1995). J. Aerosol Sci. 26, 459-475.

Ude S., \& Fernández de la Mora J. (2005). Journal Aerosol Sci., 36, 1224-1237.

Ugrinov A., Sen A., Reber A.C., Qian M., \& Khanna S.N. (2008). [Te $\left.{ }_{2} \mathrm{As}_{2}\right]^{-2}$ : A Planar Motif with

"Conflicting" Aromaticity. J. Amer. Chem. Soc., 130, 782.

Wallace W.T., Wyrwas R.B., Whetten R.L., Mitric R., \& Bonacic-Koutecky V. (2003). Oxygen Adsorption on Hydrated Gold Cluster Anions: Experiment and Theory. J.Amer. Chem. Soc., 125, 8408.

Zhang C., Yoon B., \& Landman U. (2007). Predicted Oxidation of CO Catalyzed by Au Nanoclusters on a Thin Defect-Free MgO Film Supported on a Mo(100) Surface. J. Amer. Chem. Soc., 129, 2228. 
Figure 1



Figure 1. Experimental setup 
Figure 2a

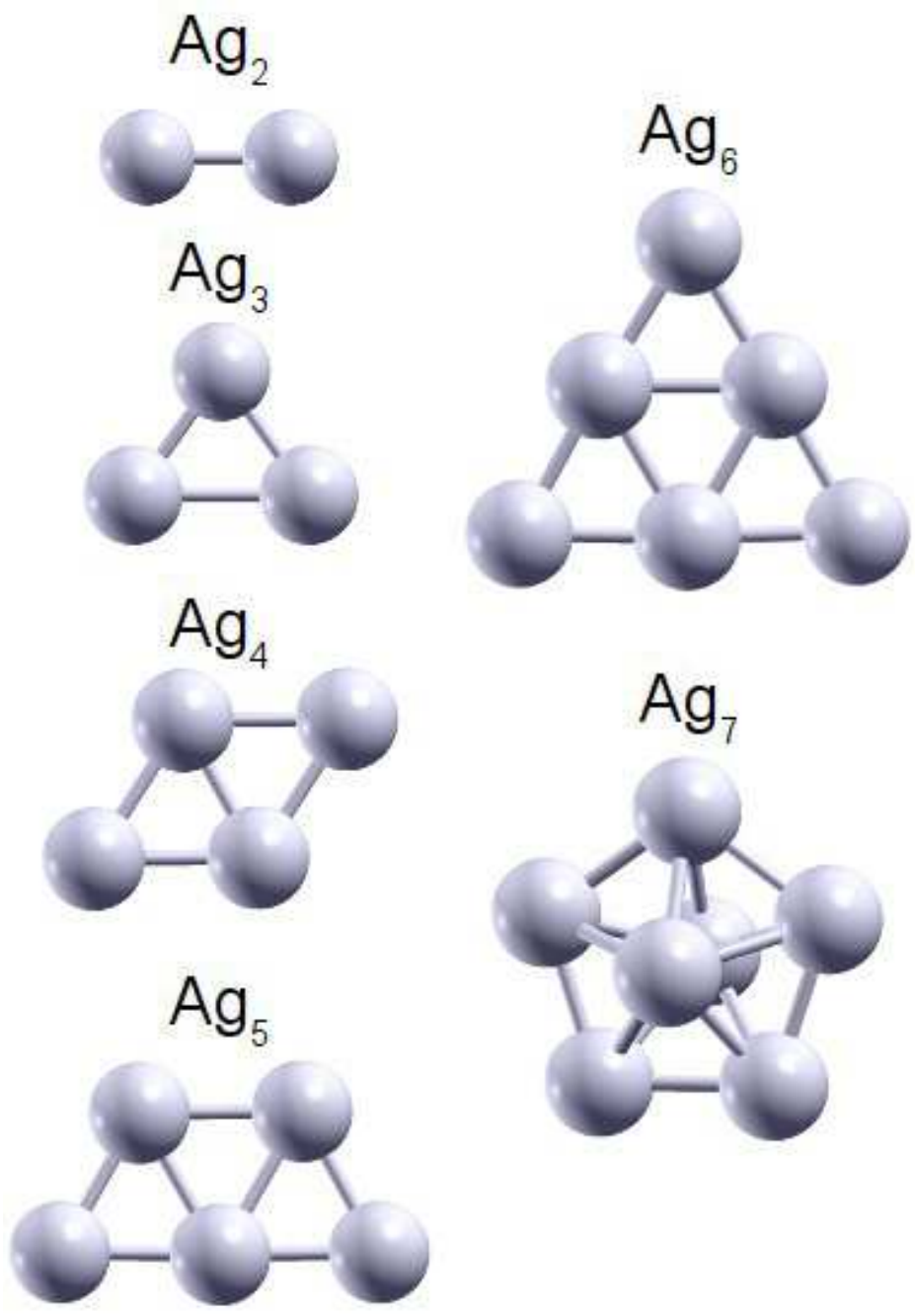

Figure 2a. The geometry of $\mathrm{Ag}_{\mathrm{n}}$ clusters, $\mathrm{n}=2-7$ 
Figure 2b


Figure $2 \mathrm{~b}$. The geometry of $\mathrm{KAg}_{\mathrm{n}}{ }^{+}$clusters, $\mathrm{n}=1-6$. 
Figure 3

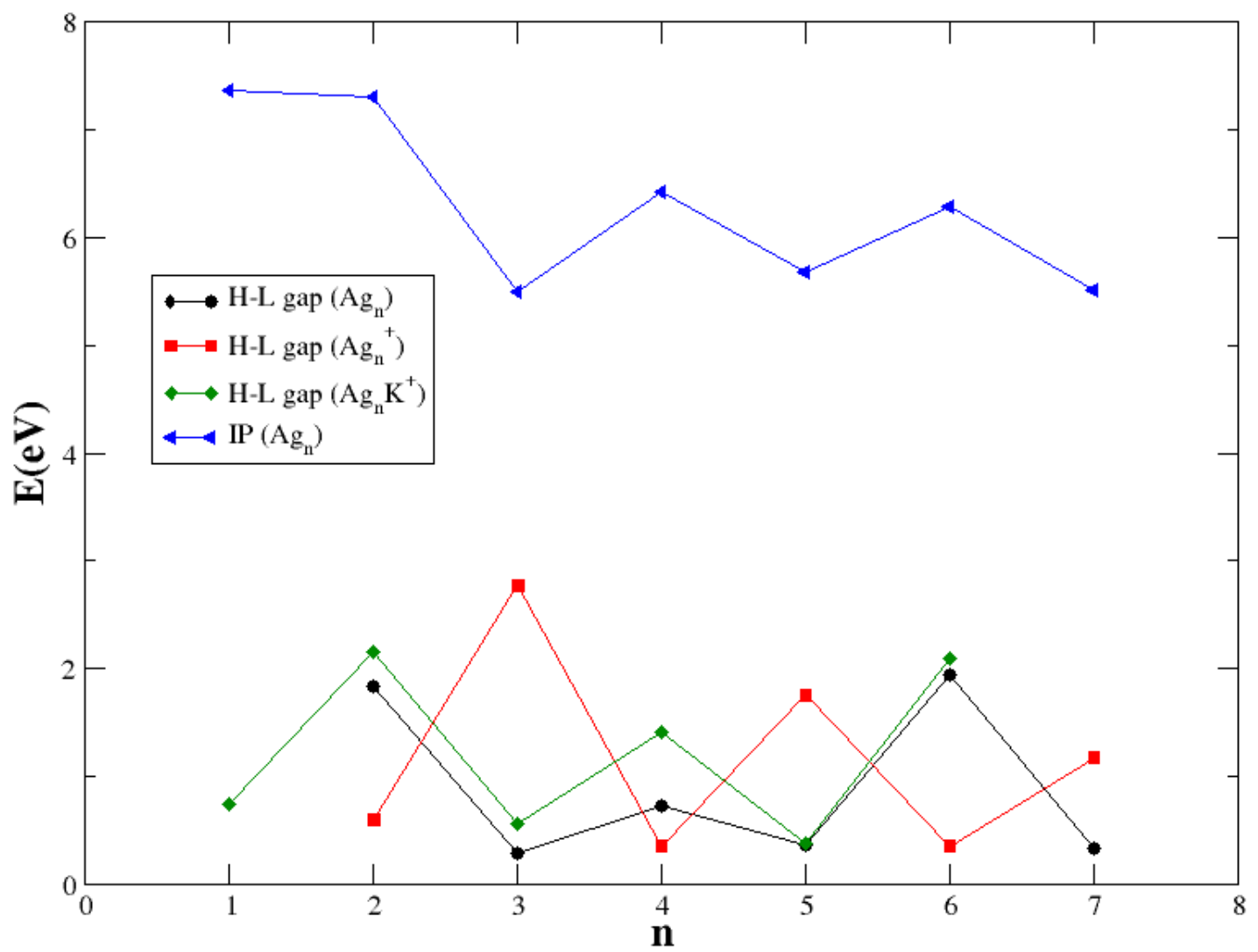

Figure 3. The HOMO-LUMO gap (H-L gap) and Ionization Potential (IP) of $\mathrm{Ag}_{\mathrm{n}}$ and $\mathrm{Ag}_{\mathrm{n}}{ }^{+}$ clusters $(n=2-7)$ and $\mathrm{Ag}_{n} \mathrm{~K}^{+}$clusters $(\mathrm{n}=1-6)$. 
Figure 4



Fig. 4 The removal energies (RE) of Ag-Ag $g_{n-1}, A g-\mathrm{Ag}_{\mathrm{n}-1} \mathrm{~K}^{+}, \mathrm{Ag}_{\mathrm{n}}-\mathrm{K}^{+}$and $\mathrm{K}-\mathrm{Ag}_{\mathrm{n}}{ }^{+}$. 
Figure 5

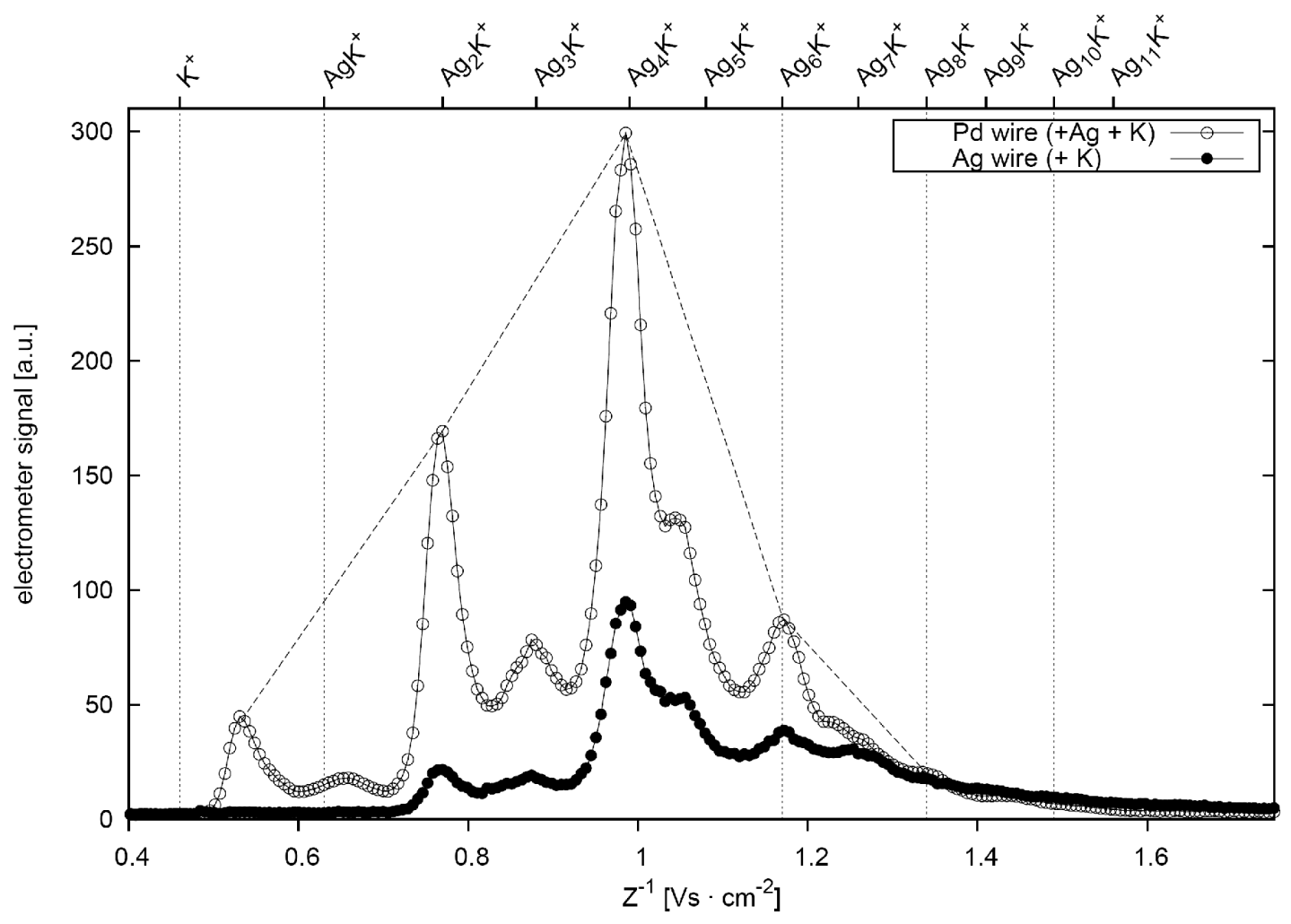

Fig. 5 Cluster size distribution in terms of the electrometer current vs. the inverse mobility $\mathrm{Z}^{-1}$. The vertical lines indicate a fit of the peak mobilities to eq. 5 . 